\title{
113 \\ Effect of TGE coronavirus strains on numbers and Ig-isotope expression of specific antibody-secreting cells from mesenteric lymph node after oral administration
}

\author{
P Berthon, S Bernard, M Olivier, E Bottreau, I Lantier, H Salmon and JM Aynaud \\ INRA Laboratoire de Pathologie Porcine, 37380 Nouzilly, France
}

Transmissible gastroenteritis (TGE) is lethal for piglets under two weeks of age but usually results in a transient diarrhea in older pigs (Hooper et al.(1966)). Caused by a coronavirus which preliminary infects intestinal epithelial cells, this swine disease can be used as model for studies on the regulation of the secretory IgA response. It is well established that the majority of IgA antibodysecreting cells (ASC) of the intestinal mucosa, before homing back to the lamina propria, transit through the mesenteric 1ymph node (MLN) where they undergo a maturation step (Mc Williams et al.(1977); Roux et al.(1981)). Mesenteric cells were isolated from adult pigs 3, 11 or 30 days after oral immunization with either a virulent (GEP-II) or two attenuated (Purdue-115, Nouzil1y) strains of coronavirus and were cocultured with the virus for one week. Responses to TGE virus were measured by enumeration of specific ASC according to the Ig-isotype ( ELISASPOT assay) from these mesenteric leukocyte cultures (Berthon et a1. (manuscript in preparation)).

Results showed that GEP-II strain, which induced diarrhea in these pigs, induced an antibody response increasing from day 3 to day 30 with a predominance of IgA ASC. In contrast, attenuated strains (no diarrhea after the viral ingestion) generated a short antibody response, decreasing from the 3 rd day after immunization and mainly represented by IgG ASC. These data support the view that the induction and the maintenance of IgA synthesis are correlated with the magnitude of the gut antigenic stimulation, with the possibility of emigration of the attenuated virus strains through the gut resulting in the stimulation of the systemic immune system. 
Hooper, B.E. and Haelterman, E.O. (1966) 'Concepts of pathogenesis and passive immunity in transmissible gastroenteritis in swine', J. Am. Vet. Med. Assoc. 149, 1580-1586.

Mc Williams, M., Phillips-Quagliata, J.M. and Lamm, M.E. (1977)

'Mesenteric lymph node B lymphoblasts which home to the smal1 intestine are precomitted to IgA synthesis', J. Exp. Med. 145, 866-875.

Roux, M.E., Mc Williams, M., Phillips-Quagliata, J.M. and Lamm, M.E. (1981) 'Differentiation pathway of Peyer's patch precursors of IgA plasma cells in the secretory immune system', Cell. Immuno1. 61, 141-143. 